\title{
Polymerized Supramolecular Assemblies
}

\author{
David F. O’Brien, * Thauming Kuo, Ulrich Liman, \\ and Henry LAMPARSKI \\ Department of Chemistry, The University of Arizona, \\ Tucson, AZ 85721 USA
}

(Received November 26, 1990)

\begin{abstract}
Supramolecular assemblies of amphiphiles include monolayers, bilayers, multilayers, as well as nonlamellar structures. The recent introduction of methods to polymerize supramolecular assemblies has increased the range of chemical and physical stabilities of these new materials. Polymerized supramolecular assemblies offer opportunities to prepare new materials for both biological and materials sciences. Polymerization has proven a valuable probe of the similarities of molecular order within bilayers and multilayers, and to examine the transformation of the first into the latter. It is also possible to initiate the transformation from lamellar to nonlamellar assemblies via polymerization induced processes within the assembly. Examples of each of these types of supramolecular conversions are described.

KEY WORDS Bilayer / Multilayer / Vesicle / Supramolecular Assembly / Photopolymerization /
\end{abstract}

Biological membranes have long been considered ordered systems. Thus our traditional instincts were rudely surprised when molecular mobility in biomembranes was exquisitely demonstrated. ${ }^{1}$ Since the early seventies the dynamic tension between order and mobility in the study of biomembranes has led to major advances in understanding those systems and to the creation of other nonbiological membrane systems. These innovations in understanding have led to the curent evolving view of a wide range of cooperative molecular systems, which are now termed supramolecular systems, and include micelles, monomolecular layers, bilayers, multilayers, nonlamellar lipid structures, as well as liquid crystals.

Biological cell membranes are composed of lipids, proteins, and carbohydrates. Cells exploit the amphiphilic nature of lipids to create anatomical boundaries, e.g. the plasma membrane and the mitochrondrial membrane. During the early 1960s researchers demonstrated that certain classes of lipids, especially glycerophospholipids, could be used to form protein-free model membranes. Both Mueller, ${ }^{2}$ who developed methods for the preparation of supported bilayer lipid membranes (BLM), and Bangham, ${ }^{3}$ who discovered that dried thin films of phospholipids spontaneously hydrate to yield selfsupported closed bilayer assemblies of several thousand lipid molecules (liposomes), are credited with this advance. The lipid bilayer in each model membrane is a two-dimensional fluid composed of lipids with their hydrophilic head groups exposed to water and their hydrophobic tails aggregated to exclude water. The bilayer structure is highly ordered yet dynamic 
due to the rapid lateral motion of the lipids within the plane of each half of the bilayer.

\section{POLYMERIZATION OF SUPRAMOLECULAR ASSEMBLIES}

Polymerization of supramolecular assemblies of lipids was first demonstrated in monolayers, vesicles, and extended bilayers. Interest in polymerizable amphiphiles dates from at least 1971, when Thomas, Drexhage, and $0^{\prime}$ Brien, ${ }^{4}$ at the Kodak Research Laboratories, demonstrated that fatty acid diacetylenes form well behaved monolayers, and that two-dimensional order is sufficient for photopolymerization of the diacetylenes. Selected diacetylenes were polymerized on the water surface or after transfer to a solid support. The polymerized monolayers were rugged enough to span electron microscope grid holes of at least $50 \mu \mathrm{m}$ diameter. An independent investigation by Day and Ringsdor $f^{5}$ showed that 10,12-nonacosadiynoic acid formed close-packed monolayers which are photopolymerizable in the solid analogous phase and yield the blue form of polydiacetylenes (PDA). Other polymerizable single chain amphiphiles include vinyl stearate, octadecyl methacrylate, octadecylfumarate, and tricosenoic acid.

The introduction of synthetic double tail amphiphiles by the Kunitake group, ${ }^{6}$ coupled with the successful demonstration of polymerization of fatty acid monolayers led directly to the next step in the early 1980s. Regen et al. (1980) described a cationic ammonium salt with a methacrylate at the end of one hydrocarbon chain. ${ }^{7}$ In short order three additional groups, Chapman, ${ }^{8}$ Ringsdorf, ${ }^{9}$ and $0^{\prime}$ Brien $^{10}$ reported the synthesis and polymeriza-tion of lipid diacetylenes in bilayers. Within a year dienoyl lipids, addi-tional methacryloyl lipids, other lipid diacetylenes, and vinyl lipids ap-peared as wel1. ${ }^{11}$ The polymerization of these reactive lipids in a self-organized array of several thousand lipid molecules into a structure that contains several polymer chains is termed the formation of polymerized vesicles (1iposomes).

The successful polymerization of lipid monolayers and bilayers contrasts with the inability to effectively polymerize single chain amphiphiles in micelles. ${ }^{12}$ The significant difference between the two systems is the greater rate of exchange for monomers from micellar than from bilayer assemblies. In micelles the exchange rate is generally competitive with the rate of polymerization, whereas in bilayer and monolayer structures the exchange rate is too slow to significantly compete with the polymerization reaction.

At least three experimental strategies can now be identified for the formation of polymerized supramolecular assemblies.

- A major approach to polymerized bilayers and other supramolecular asemblies is the preparation of polymerizable lipid monomers, the formation of the lipid assembly from the monomer, and the subsequent chain polymerization of the monomers in the assembly. A variation on this approach is to incorporate reactive moieties that allow condensation polymerization of the assembly.

- Alternatively, the amphiphile is polymerized in isotropic solution and then the supramolecular assembly is formed from the prepolymerized amphiphiles. This approach has been extensively studied in monolayers, as well as liquid crystals.

- Finally, monomers or polymers may be associated with preformed assemblies. Polymerizable species may be electrostatically associated with the amphiphile either before or after assembly formation (polymerizable 
gegions), and polymerized in the assembly. Polymers and oligomers may be adsorbed or anchored onto the surface of membrane assemblies.

Polymerizable amphiphiles are prepared by introduction of the reactive group into different regions of the lipid molecule. The polymerizable group may be at the chain terminus in the middle of the bilayer, near the middle of the hydrophobic chains, attached to the hydrophilic head group, or electrostatically associated with a charged lipid. The first two locations have no direct influence on the membrane water interface, whereas the mobility of the lipid chains is significantly decreased by polymerization in these systems. In contrast, the last two methods alter the membrane water interface but have less effect on the hydrophobic interior of the membranes. If there is only one reactive group per lipid linear polymer chains are formed in supramolecular assemblies. The presence of a second polymerizable group per molecule allows crosslinking of the polymer chains. A host of reactive moieties may be utilized to modify the above lipid classes to make them polymerizable. These groups include diacetylene, acryloyl, methacryloyl, itaconyl, dienoyl, muconyl, styryl, vinyl, thiol, and chain terminal isocyanates.

A variety of transformations or conversions of one supramolecular assembly into another have appeared in the literature. Although the new methods of polymerizing these assemblies are usually associated with the stabilization of the assembly, it is also clear that these two-dimensional polymerizations are valuable for the characterization of supermolecular conversions or for the initiation of supramolecular morphology changes.

\section{CONVERSION FROM BILAYER VESICLES TO MULTILAYER FILMS}

Hydrated lipid bilayer membranes can be formed by the hydration of a thin film of lipids. The nature of the lipid film is largely unknown, but is usually assumed to be amorphous because the film is formed by the evaporation of the organic solvent of the lipid. Once the water is added to the lipids, they self-assemble into supramolecular structures that are dictated by the molecular forces, e.g. hydrophobic effect, hydrogen bonding, dipolar interactions. The question arises whether the organized assembly can be maintained or transformed into another assembly as the water content is diminished in a controlled manner. Kunitake and coworkers have recently reported that bilayer vesicles may be conveniently converted to multilayer films by such a process. ${ }^{13-15}$ An aqueous dispersion of bilayer vesicles was spread on a solid support and then allowed to dry slowly to yield a dried film. Examination of the dried film by X-ray scattering (edgeon) and differential scanning calorimetry indicated the presence of bilayer (lamellar) structures. In order to evaluate the completeness of retention of lamellar lipid arrangements as the hydrated bilayers are converted into dried multilayers, we have designed and synthesized new diacetylenic lipids. ${ }^{16-18}$

These new materials allow evaluation of the efficiency of polydiacetylene (PDA) formation in both bilayers and the multilayer films (cast films). The formation of PDAs is a useful test of the lamellar nature and the molecular order within an assembly, because the polymerization reaction is especially sensitive to themolecular packing of the monomers. Monolayers of diacetylenic fatty acids are only polymerizable in the condensed phase. ${ }^{5}$ Bilayers of lipid diacetylenes are polymerizable in the solid-analogous phase, that is at temperatures below the lipidphase transition temperature between the $L_{\beta}$ and $L_{\alpha}$ phases. ${ }^{19,20}$ At higher temperatures the lipid chain 
disordering inhibits the topotactic polymerization reaction. It is also recognized that the disordering of lipid chains, which occurs upon the formation of small sonicated bilayer vesicles, hinders the formation of PDA. ${ }^{20}$ Therefore the successful formation of PDAs in large vesicles and in cast multilayer films prepared from them would inciate the retention of an ordered lamellar structure. Furthermore the absorption maxima of PDAs is indicative of the polymer chain length and/or any polymer conformation that decrease the effective conjugation length.

Four lipid diacetylenes based on a glutamate backbone are shown below. The complete syntheses and structural characterization of these new molecules is described elsewhere. ${ }^{21}$ Each of these lipids forms bilayers upon hydration as shown by their lipid phase transition due to cooperative lipid chain behavior. Both hydrophobic effects due to the lipid chains and the hydrogen bonding of the amide linkage provide the driving force for the formation of the supramolecular assembly.

The variations in the lipid structure are limited to the head group in order to keep the diacetylenic chains constant. The head group structures were selected to vary the lipid phase transition temperature $\left(T_{m}\right)$ from below to above room temperature. The $T_{m}$ of bilayers of 1 was at $18{ }^{\circ} \mathrm{C}$. If the spacer length between the triethylammonium and the glutamate backbone was increased from five methylenes (1) to ten methylenes (2), the $T_{m}$ was increased to $30.5{ }^{\circ} \mathrm{C}$. It is also possible to increase the $T_{m}$ by changing the quaternary ammonium substitution from ethyl (1) to methyl (3). The $\mathrm{T}_{\mathrm{m}}$ for hydrated bilayers of 3 was $26.0^{\circ} \mathrm{C}$. Finally, the incorporation of a phenoxy group into the spacer (4) increases the $T_{m}$ to $35{ }^{\circ} \mathrm{C}$.
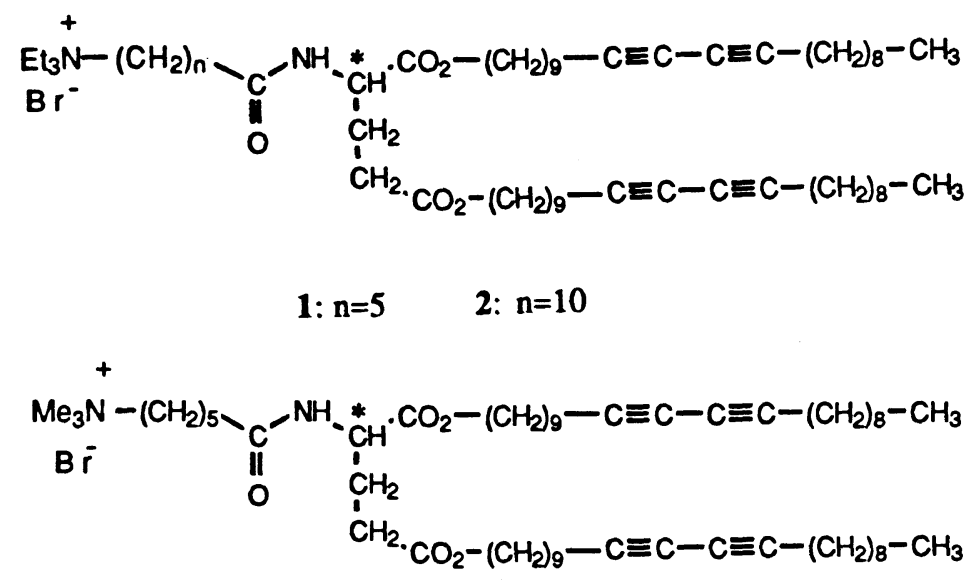

3

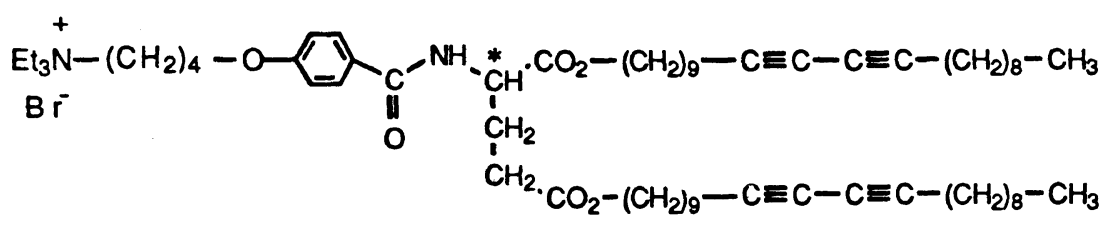


Bilayer membranes of each glutamate lipid were prepared by hydration (Milli-Q water) of a thoroughly dry thin lipid film, followed by vortexing and only mild sonication. The lipid bilayers of either lipid 2, 3 , or 4 were photosensitive at $22{ }^{\circ} \mathrm{C}$ to $254 \mathrm{~nm}$ light, and each rapidly formed purple-blue PDA. In contrast hydrated bilayers of lipid 1 did not form highly colored PDA when irradiated with $254 \mathrm{~nm}$ light at room temperature. However when bilayers of 1 were cooled with ice water the formation of purple-blue PDA was readily observed. The absorption maxima of the PDAs formed from these lipid bilayers are given in Table $I$, and the absorption spectrum of cast mutilayers of 4 is shown in Figure 1.

Table I. Absorption maxima (nm) of the polydiacetylenes formed in lipid bilayer membranes and in cast multilayer films.

\begin{tabular}{ccc} 
Lipid & \multicolumn{2}{c}{ Absorption Maxima } \\
& Bilayer & Cast Film \\
1 & 640 & 610 \\
2 & 610,570 & 610,570 \\
3 & 610,570 & 610,570 \\
4 & 590 & 590
\end{tabular}

Cast multilayer films were prepared at room temperature from unpolymerized bilayers of each of the lipids. The hydrated diacetylenic lipid bilayers were deposited on a clean glass slide. After drying over two days at constant temperature and humidity, the resultant colorless thin transparent films were vacuum dried. Exposure of film samples of each lipid to $254 \mathrm{~nm}$ light at room temperature immediately caused the films to become colored. The uniform purple-blue color of the films intensified with exposure time in the manner associated with PDA formation. The absorption maxima of the PDAs formed in the cast films are also shown in Table I. It

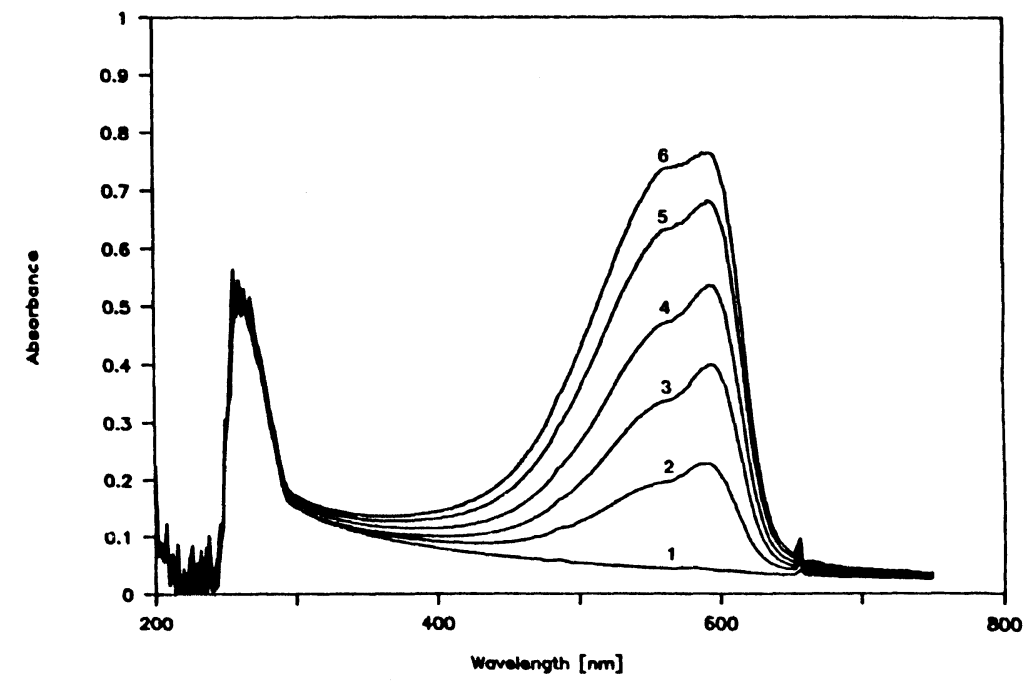

Figure 1. Absorption spectra of a cast multilayer film of lipid 4. The spectra were recorded after exposure to $254 \mathrm{~nm}$ light for the indicated times: curve $1,0 \mathrm{~s} ; 2,10 \mathrm{~s} ; 3,30 \mathrm{~s} ; 4,60 \mathrm{~s} ; 5,120 \mathrm{~s} ; 6,180 \mathrm{~s}$. 
is readily apparent that that the PDAs formed in bilayers and films of lipids 2, 3 , and 4 have very similar absorption properties. Cast films from each of these three lipids also show reversible thermochromic behavior between $22^{\circ} \mathrm{C}$ and $50{ }^{\circ} \mathrm{C}$.

In contrast, the PDA formed in the cast multilayers of 1ipid 1 shows an absorption maxima at distinctly shorter wavelength than is observed for the bilayers of this lipid. This shift of the absorption maxima suggests that the polymer chain lengths of PDA formed in cast multilayers of 1 is shorter than that formed in the original bilayers. Although the effect is smal1, it nevertheless draws our attention to the fact that not all of these diacetylenic lipids behave in the same manner when lipid bilayers are converted into cast multilayers. The major difference between bilayers of 1 and of $2-4$ is the position of the $T_{m}$ relative to room temperature. This difference may be crucial during the cast multilayer film dehydration procedure. The $T_{m}$ of lipid bilayers shifts to higher temperature as the water content is decreased. ${ }^{21}$ Therefore the $T_{m}$ of 1ipids 2-4 will become even greater than room temperature. However in the case of 1 , whose $T_{m}$ is below room temperature, the dehydration increases the $\mathrm{T}_{\mathrm{m}}$ and the bilayers undergo a transition from the $\mathrm{L}_{\alpha}$ to the $\mathrm{L}_{\beta}$ phase. This dehydration induced excursion of the phase transition could lead to disordering of the cast film layers, which is reflected in the observed PDA absorption spectrum.

The successful conversion of bilayer membranes to cast multilayer films provides an alternative method to the classical Langmuir-Blodgett technique for the preparation of multilayer films. The well known L-B techniques are currently widely used for the formation of well-ordered multilayers of amphiphiles for scientific studies and for potential applications in photonic and electronic materials. These studies indicate, that with proper attention to the lipid phase transition temperature, the conversion of liposomes to cast multilayer films can be a convenient alternative to the L-B methodology for the formation of repetitive multilayer assemblies.

\section{CONVERSION FROM BILAYER TO NONBILAYER SUPRAMOLECULAR STRUCTURES}

The partial polymerization of a hydrated bilayers composed of a single polymerizable lipid yields domains of polymerized bilayer and residual monomer. The polymerization of a two component systems, with one polymerizable and one nonpolymerizable component, can result in phase separation and domain formation as we11. ${ }^{22,23}$ The polymerizable lipids form covalentlylinked domains as the reaction proceeds, which in turn produces domains of the nonpolymerizable component. If both components prefer a bilayer (1ame1lar) structure, then vesicles of the two components will retain their shape and integrity. Recently Frankel et al. showed that if the nonpolymerizable component prefers a nonbilayer rather than a lamellar structure, the membrane will be destabilized. 24

Certain 1ipids, e.g. phosphatidylethanolamines (PE), are of particular interest because they form nonbilayer structures under physiological conditions. ${ }^{25}$ These nonlamellar structures are usually the inverted hexagonal phase $\left(\mathrm{H}_{\mathrm{II}}\right)$ and/or an isotropic phase, e.g. inverted cubic phase ( $\left.\mathrm{Q}_{\mathrm{II}}\right)$. Bilayer vesicles from pure $P E$ are only stable in buffer solutions above $\mathrm{pH}$ 9.0, where the $P E$ is negatively charged. ${ }^{26}$ If $P E$ is combined with a bilayer forming lipid, e.g. phosphatidylcholine (PC), stable bilayer vesicles are formed at $\mathrm{pH} 7$. Processes that lead to phase separation of the $\mathrm{PC}$ and $\mathrm{PE}$ can trigger the PE lamellar to nonlamellar transition and thereby destabil- 
ize the vesicle membrane. Here we describe the photopolymerization induced conversion of a lamellar to a nonlamellar supramolecular assembly.

Consider the properties of two-component vesicles of dioleoylPE (DOPE) and a polymerizable PC (SorbPC). The vesicles are stable prior to polymerization and can encapsulate aqueous markers, e.g. the fluorescent dye calcein. Physical or chemical processes that disrupt the bilayer character of the vesicle will cause the release of these aqueous markers. For example, treatment of the vesicles with a detergent will convert the bilayer into mixed micelles and release the aqueous contents. Thus an increase in vesicle permeability is indicative of a bilayer destabilization process which may be due to the conversion from a bilayer to a nonbilayer structure. The SorbPC (5) contains a photosensitive sorbyl moiety (absorption maxima in water - $257 \mathrm{~nm}$ ) at the terminal end of each acyl chain, and it is readily photopolymerized by exposure to $254 \mathrm{~nm} 1$ ight.

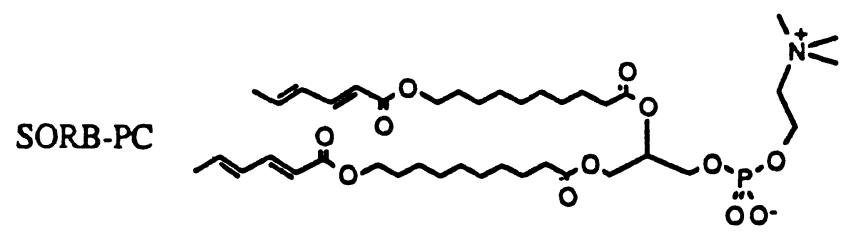

The vesicle permeability of DOPE/SorbPC ( $2 / 1$ and $3 / 1$ mole ratios) vesicles was determined as a function of the percent conversion of monomeric SorbPC to polymerized lipid. An increase in membrane permeability results in increased calcein fluorescence, which was related to percent leakage. Figure 2 shows the membrane permeability to calcein for vesicles composed of a) dioleoyl PE/SorbPC (2/1); b) TPE/SorbPC (2/1) (TPE is trans phosphatidylated eggPC); and c) dioleoyl PC/SorbPC (2/1). The first two are mixtures of $\mathrm{PE}$ and polymerizable $\mathrm{PC}$, and the last is a control membrane

\section{CALCEIN LEAKAGE}

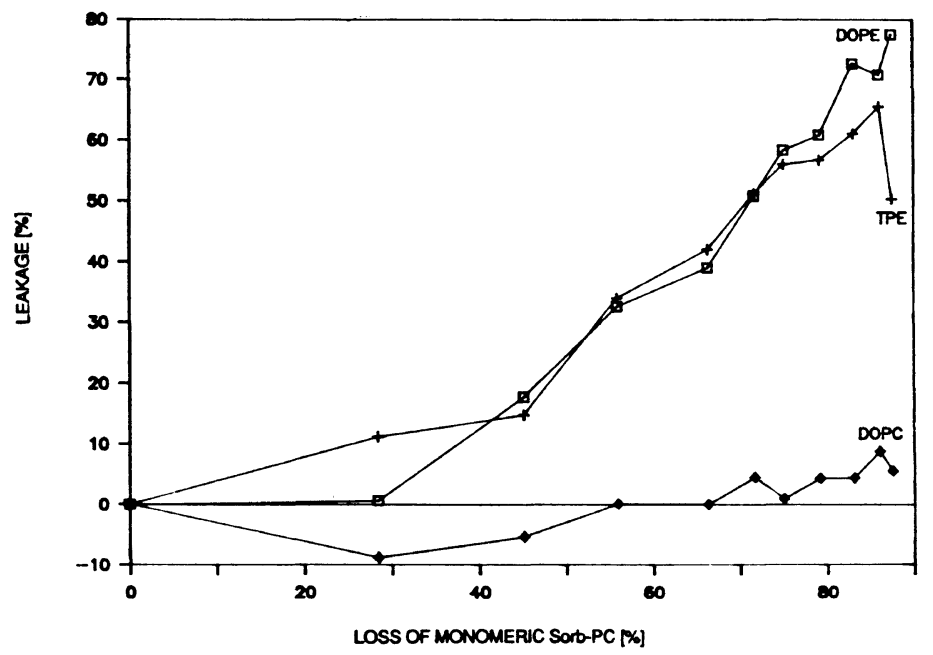

Figure 2. Leakage of calcein at $24^{\circ} \mathrm{C}$ from vesicles vs. the loss of monomeric SorbPC during the photopolymerization. The leakage was calculated from an increase in fluorescence intensity. Vesicles were prepared in a $2 / 1$ molar ratio of DOPE/SorbPC, TPE/SorbPC, and DOPC/SorbPC and desalted with $150 \mathrm{mM} \mathrm{NaC1}, 2 \mathrm{mM}$ imidazole, $2 \mathrm{mM}$ TES buffer at $\mathrm{pH} 7.4$. 
composed of a PC and polymerizable PC. As seen in Figure 2, a significant release of calcein is observed after 20 to 308 (1-2 min irradiation) loss of monomeric SorbPC, but only if the liposomes contain PE. Irradiation of the control membranes of DOPC/SorbPC did not increase the membrane permeability even after greater than $80 z$ loss of monomer.

The vesicle destabilization appears to require the presence of multior oligolamellar vesicles. Experiments similar to those shown in Figure 2 do not show a change in vesicle permeability if vesicles are unilamellar. ${ }^{27}$ The leakage of oligolamellar vesicles requires the following processes: 1) lateral phase separation of the lipids into enriched domains; 2) bilayer contact between apposed bilayer surfaces; and 3) the ability of the contacting lamellae to form nonlamellar structures. All three conditions are met in the case of the oligolamellar vesicles of partially polymerized PE/SorbPC.

Photopolymerization of PE/SorbPC causes phase separation and produces enriched domains of PE. Since PE surfaces are less strongly hydrated than PC or PC/PE membranes, ${ }^{28}$ this process will allow the close approach of the enriched PE domains in apposed bilayers. Siege ${ }^{29}$ has proposed a kinetic model for the transition from lamellar to nonlamellar lipid phases. Contact between the bilayers leads to the formation of inverted micellar intermediates, which can partition between inverted hexagonal $\left(\mathrm{H}_{\mathrm{II}}\right)$ and inverted cubic $\left(Q_{I I}\right)$ lipid structures. This partition is controlled by the balance of forces between the lipid chains and the head group, which can be analyzed in terms of the spontaneous radius of curvature of the lipids. ${ }^{30}$ The path-way to the $\mathrm{Q}_{I I}$ phase proceeds through an interlamellar attachment (ILA), which is an hourglass shaped attachment between the two original lamellae. The ILA effectively fuses the lamellae. If this occurs in a oligolamellar vesicle, then an aqueous permeability pathway is created between the in-terior and exterior of the vesicle.

An ILA is an isotropically symmetric structure, therefore in sufficient concentration it gives rise to an isotropic NMR signal. Examination of extended bilayers of unexposed DOPE/SorbPC by ${ }^{31} \mathrm{P}-\mathrm{NMR}$ shows a typical lamellar powder pattern at room temperature. ${ }^{31}$ As the sample temperature is increased the sample begins to display an isotropic signal overlaid on the lamellar powder pattern. Photopolymerization of additional samples of the same lipids causes a decrease in the temperature for the appearance of the isotropic signal. Polymerization of the membranes decreased the phase transition temperature from a lamellar to a nonlamellar isotropic structure. The properties of this isotropic structure are the same as those ascribed to ILAs from observations on other 1ipid systems.

The formation of ILAs are predicted to mediate the fusion of vesicles with one another or precede the formation of the $Q_{I I}$ phase. ${ }^{29}$ The experiments described here indicate that ILAs may also mediate the leakage of the aqueous contents from oligolamellar vesicles via the fusion of bilayers within a single vesicle. The ability of light to penetrate the bilayers and initiate polymerization induced phase separation in each of the lamellae of the vesicle is an important aspect of this conversion process. The wellknown methods of controlling the spatial and temporal delivery of light endow this photopolymerization induced transformation of lamellar structures with important capabilities for the release of chemical and biological reagents. 
Acknowledgement is made to the donors of the Petroleum Research Fund, administered by the American Chemical Society, for partial support of this research, and to the National Science Foundation.

\section{REFERENCES}

1. M. Edidin in "Membrane Structure", eds. J. B. Finean and R. H. Miche11, Elsevier, New York, N.Y., 1981, pp. 37-82.

2. P. Mueller, D. O. Rudin, H. T. Tien, and W. C. Westcott, Nature 194, 979 (1962).

3. A. D. Bangham and R. W. Horne, J. Mol. Biol. 8, 660 (1964).

4. H. T. Thomas, K. H. Drexhage, and D. F. O'Brien, Eastman Kodak Research (1971).

5. D. R. Day and H. Ringsdorf, J. Polym. Sci. Polym. Lett. Ed. 16, 205 (1978).

6. T. Kunitake and Y. Okahata, J. Am. Chem. Soc. 99, 3860 (1977).

7. S. L. Regen, B. Czech, and A. Singh, J. Am. Chem. Soc. 102, 6638 (1980).

8. D. S. Johnston, S. Sanghera, M. Pons, and D. Chapman, Biochim. Biophys. Acta 602, 57 (1980).

9. H. Hub, B. Hupfer, H. Koch, and H. Ringsdorf, Angew. Chem. Int. Ed. Eng1. 19, 938 (1980).

10. D. F. O'Brien, T. H. Whitesides, and R. T. Klingbiel, J. Polym. Sci. Polym. Lett. Ed. 19, 95 (1981).

11. Reviewed in D. F. O'Brien and V. Ramaswami, "Encyclopedia of Polymer Science and Engineering", second edition, vol. 17, eds. H. Mark, N. Bikales, C. Overberger, and J. Menges, John Wiley \& Sons, New York, N.Y., 1989, pp. 108-135; and in H. Ringsdorf, B. Schlarb, and J. Venzmer, Angew. Chem. Int. Ed. Engl. 27, 113 (1988).

12. S. Hamid and D. Sherrington, J. Chem. Soc. Chem. Commun., 936 (1986).

13. M. Shimomura, R. Ando, and T. Kunitake, Ber. Bunsenges. Phys. Chem. 87,1134 (1983).

14. T. Kunitake, M. Shimomura, T. Kajiyama, A. Harada, K. Okuyama, and M. Takayanagi, Thin Solid Films 121, L89 (1984).

15. N. Nakashima, M. Kunitake, T. Kunitake, S. Tone, and T. Kaliyama, Macromolecules 18, 1515 (1985).

16. T. Kuo and D. F. O'Brien, J. Am. Chem. Soc. 110, 7571 (1988).

17. T. Kuo and D. F. O'Brien, Macromolecules 23, 3225 (1990).

18. T. Kuo and D. F. O'Brien, J. Chem. Soc. Chem. Commun., 839 (1990).

19. E. Lopez, D. F. O'Brien, and T. H. Whitesides, J. Am. Chem. Soc. 104, 305 (1982).

20. T. Kuo and D. F. O'Brien, Langmuir 7, in press (1991).

21. D. Chapman in "Form and Function of Phospholipids" second edition, eds. G. B. Anse11, J. N. Hawthorne, and R. M. C. Dawson, Elsevier Scientific Publishing Co., Amsterdam, 1973, pp. 117-142.

22. H. Gaub, E. Sackmann, R. Buschl, and H. Ringsdorf, Biophys. J. 45, 725 (1984).

23. P. N. Tyminski, L. H. Latimer, and D. F. O'Brien, J. Am. Chem. Soc. 107,7769 (1985).

24. D. A. Franke1, H. Lamparski, U. Liman and D. F. O'Brien, J. Am. Chem. Soc. 111,9262 (1989).

25. J. M. Seddon, Biochim. Biophys. Acta 1031, 1 (1990).

26. J. G. Strollery and W. J. Vail, Biochim. Biophys. Acta 471, 372 (1977). 
27. H. Lamparski, U. Liman, D.A. Franke1 and D.F. O'Brien, Biochemistry, in press.

28. V. A. Parsegian, N. Fuller and R. P. Rand, Proc. Nat1. Acad. Sci. U.S.A. 76, 2750 (1979).

29. D. P. Siege1, Biophys. J. 49, 1155 (1986); and 1170 (1986).

30. S. M. Gruner, Proc. Nat1. Acad. Sci. U.S.A. 82, 3665 (1985).

31. J. A. Barry, U. Liman, M. F. Brown, and D. F. O'Brien, Biophys. J. 55,340 a (1989). 